\title{
Treatment of Gram-negative pneumonia in the critical care setting: is the beta-lactam antibiotic backbone broken beyond repair?
}

\author{
Matteo Bassetti ${ }^{1 *}$, Tobias Welte ${ }^{2}$ and Richard G. Wunderink ${ }^{3}$
}

\begin{abstract}
Beta-lactam antibiotics form the backbone of treatment for Gram-negative pneumonia in mechanically ventilated patients in the intensive care unit. However, this beta-lactam antibiotic backbone is increasingly under pressure from emerging resistance across all geographical regions, and health-care professionals in many countries are rapidly running out of effective treatment options. Even in regions that currently have only low levels of resistance, the effects of globalization are likely to increase local pressures on the beta-lactam antibiotic backbone in the near future. Therefore, clinicians are increasingly faced with a difficult balancing act: the need to prescribe adequate and appropriate antibiotic therapy while reducing the emergence of resistance and the overuse of antibiotics. In this review, we explore the burden of Gram-negative pneumonia in the critical care setting and the pressure that antibiotic resistance places on current empiric therapy regimens (and the beta-lactam antibiotic backbone) in this patient population. New treatment approaches, such as systemic and inhaled antibiotic alternatives, are on the horizon and are likely to help tackle the rising levels of beta-lactam antibiotic resistance. In the meantime, it is imperative that the beta-lactam antibiotic backbone of currently available antibiotics be supported through stringent antibiotic stewardship programs.
\end{abstract}

\section{The 'antibiotic backbone'}

Antibiotics for the treatment of serious bacterial infections have immeasurable benefits for the critically ill patient and have greatly reduced morbidity and mortality since their widespread adoption in the 1950s. However, global overuse of these drugs has led to the development of resistance and decreased effectiveness, making it increasingly difficult to choose appropriate antibiotic therapy options for lifethreatening infections, such as pneumonia, in the intensive care unit (ICU).

The term 'antibiotic backbone' was coined to describe those drugs that form the foundation of antimicrobial therapy; these backbone antibiotics treat most bacterial infections effectively, and appropriate empirical use reduces both mortality and the emergence of resistance [1-3]. The choice of antibiotics is based on many factors, including recommendations from guidelines [4-6] and evidencebased reviews [1, 7]. Currently, the antibiotic backbone

\footnotetext{
* Correspondence: bassetti.matteo@aoud.sanita.fvg.it

'Santa Maria Misericordia University Hospital, Piazzale S. Maria Misericordia

15, 33100 Udine, Italy

Full list of author information is available at the end of the article
}

for the treatment of pneumonia consists of drugs from the beta-lactam class. However, increasing resistance rates are beginning to seriously limit the clinical utility of beta-lactams $[8,9]$. Therefore, in this review, we explore the current use of beta-lactam antibiotics, the challenges posed by increasing resistance rates, and how the betalactam antibiotic backbone might be strengthened in the future. The article reflects our best knowledge and shared professional opinion of the currently available evidence on the topics discussed, but an exhaustive or systematic review of the literature would be outside of its scope.

\section{Pneumonia in the intensive care unit Burden and mortality}

Hospital-acquired pneumonia (HAP) is one of the most common infections in the ICU $[10,11]$; published rates ranged from five to more than 20 cases per 1000 hospital admissions [11, 12]. HAP is also associated with high morbidity and mortality as well as a high health-care and economic burden [13]. Up to $44 \%$ of all HAP infections are acquired in the ICU; of these, up to $90 \%$ are ventilator- 
associated pneumonia (VAP) [12, 14]. VAP is currently defined as pneumonia arising more than 48-72 hours after endotracheal intubation [4]. However, diagnosis of HAP/ VAP may be subjective and open to interpretation. The resulting considerable variation in reporting makes comparison of data between institutions difficult [15]. Indeed, the magnitude in variability shows that current surveillance definitions of VAP perform poorly in the clinical setting and suggests that a new, more objective definition is required [15]. Regardless of criteria for diagnosis, VAP is associated with poor clinical outcomes, including increased morbidity and mortality $[12,16]$, increased hospital stay, and increased duration of mechanical ventilation [17]; notably, the duration of ventilation is an important risk factor in the development of VAP [18].

In addition to recognizing HAP and VAP, current guidelines recognize health care-associated pneumonia (HCAP); this classification includes patients with pneumonia who were recently hospitalized in an acute care hospital, resided in a nursing home or long-term care facility, or received recent intravenous (IV) antibiotic therapy [4]. Many of these patients have bacterial etiologies that require the same broad-spectrum beta-lactam-based treatment as HAP/VAP.

Mortality rates for HAP/VAP are influenced by many factors, such as patient age, presence of underlying comorbidities, adequacy of antibiotic treatment given, and, for those with VAP, time on mechanical ventilation [17]. Mortality rates vary from study to study, but up to one third of all HAP-related deaths are directly attributable to pneumonia [19]. Crude mortality rates for VAP range from $24 \%$ to $50 \%$ but can reach up to $76 \%$ in specific settings with high-risk pathogens [20]. Attributable mortality rates for VAP are difficult to determine but may be less than $10 \%[12,16,21]$, given that VAP occurs in patients who have already suffered a critical illness.

\section{Etiology}

The distribution of pathogens that comprise the etiology of pneumonia (including HAP, VAP, and HCAP) varies from region to region [22, 23]. The 2009-2012 SENTRY antimicrobial surveillance program included data from nearly 13,000 isolates from patients hospitalized with pneumonia, collected from 53 hospitals across the USA and Europe. The same top 11 organisms were observed in both geographic regions, albeit in different rank orders (Fig. 1); the most frequently observed Gram-positive pathogen was Staphylococcus aureus, whereas the most common Gram-negative pathogen was Pseudomonas aeruginosa. Other common Gram-negative pathogens included Klebsiella spp., Enterobacter spp., Acinetobacter spp., and Escherichia coli [23].

\section{The antibiotic backbone under strain and the rise of resistance}

To treat pneumonia in the ICU, current guideline recommendations focus on the initiation of effective and timely empiric antibiotic therapy combined with resuscitation and supportive measures. Beta-lactam antibiotics alone or as part of a combination regimen are the mainstay of empirical antibiotic guideline recommendations [4-6]. Despite the availability of beta-lactam treatment options, clinical cure rates for HAP/VAP rarely exceed $60 \%$, and recurrence rates are high [24-26]. The beta-lactam antibiotic backbone is under strain from the increased prevalence and variety of bacterial antibiotic resistance. The frequency of multi-drug-resistant (MDR) pathogens has accelerated dramatically in recent years (Fig. 2) [27-29]. The increasing frequency and variety of resistance patterns have also necessitated the introduction of definitions for 'extensively drug-resistant' pathogens (nonsusceptibility to at least one agent in all but two or fewer antimicrobial categories) and 'pandrug-resistant' pathogens

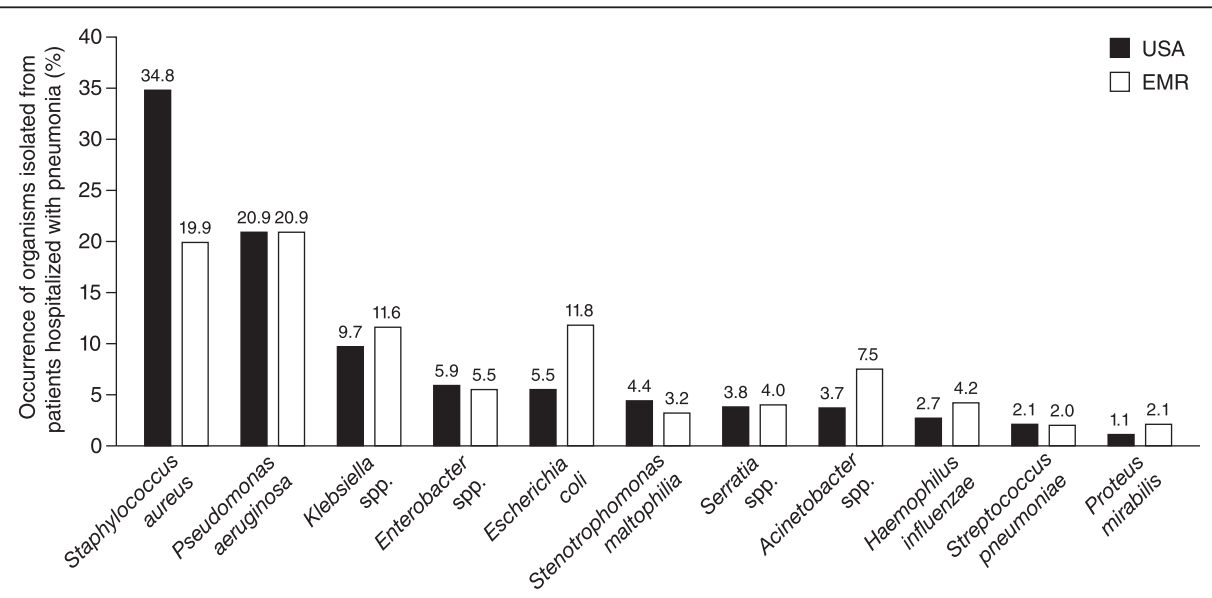

Fig. 1 Etiology of pneumonia in the hospital and critical care setting. EMR Europe and Mediterranean Region. Data from Sader et al. [23] (2014) 


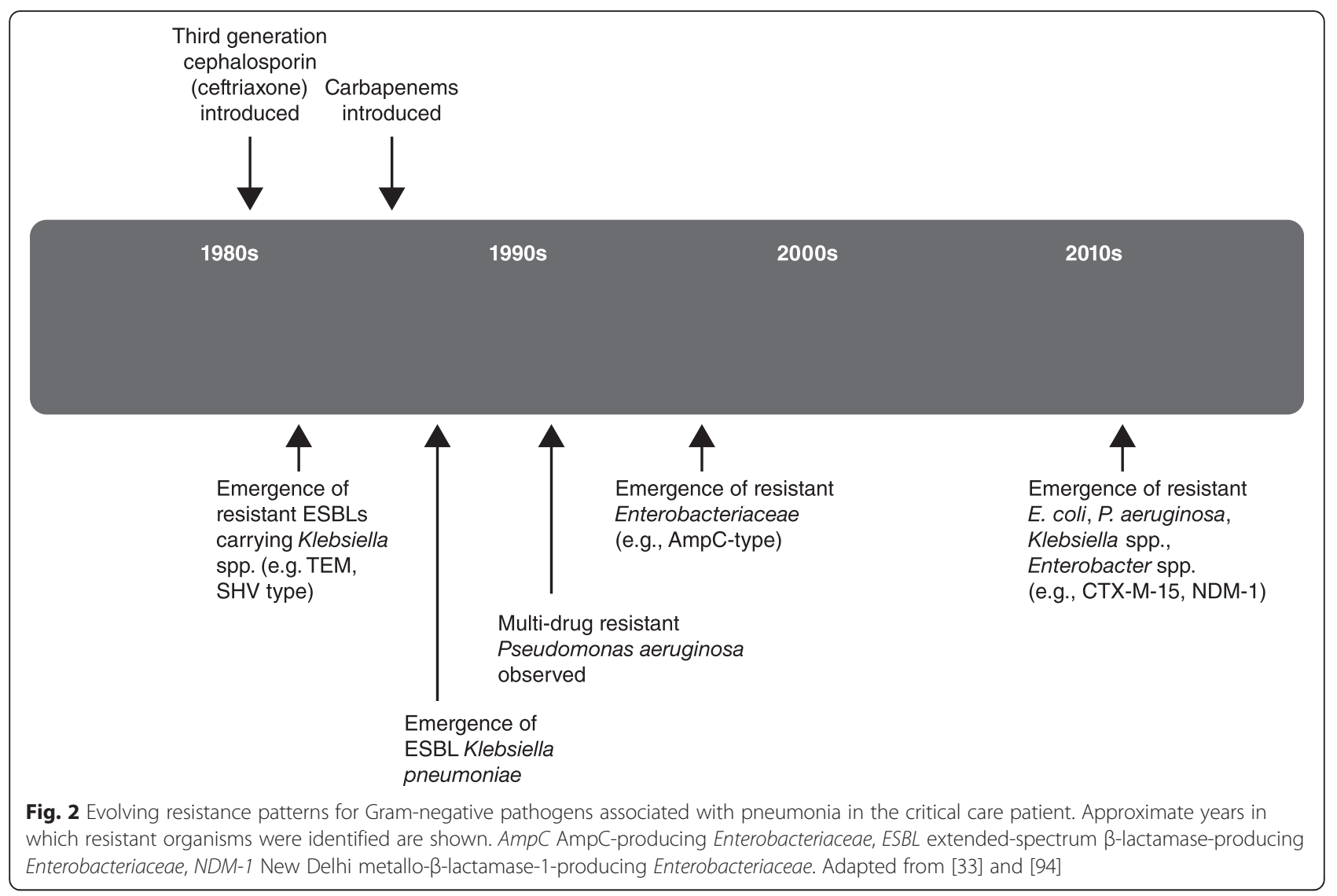

(non-susceptibility to all agents in all antimicrobial categories) [30].

Rates and patterns of antibiotic resistance differ between countries, regions, and hospitals [22, 23, 31], and high levels of resistance have been observed across all main strains of pathogens. For example, the SENTRY study reported reduced susceptibility of $P$. aeruginosa to most antimicrobials tested, including ceftazidime (79.6/68.7 \% susceptibility in USA/Europe), meropenem (76.3/65.8 \%), and piperacillin/tazobactam (72.9/63.9 \%). Furthermore, Klebsiella spp. showed extended-spectrum beta-lactamase (ESBL) phenotype prevalences of $19.5 \%$ and $35.1 \%$ in the USA and Europe, respectively; meropenem was active against $62.3 \%$ and $78.7 \%$ of ESBL Klebsiella spp. [23]. These concerning levels of beta-lactam resistance in Gram-negative pathogens are reflected in a recent comprehensive report issued by the World Health Organization (WHO), which included data from 129 WHO member states on nine bacteria-antibacterial drug combinations [22]; the report included global resistance data on a number of Gram-positive and Gram-negative pathogens that are commonly involved in pneumonia (Table 1). WHO member states reported high rates of beta-lactam resistance, including resistance to third-generation cephalosporins and carbapenems in K. pneumoniae [22]. In addition to these resistance mechanisms, new mechanisms-e.g., New Delhi metallo-beta-lactamases (NDM-1 and NDM-4)-are emerging [32]. Given the global increase in antibiotic resistance rates and the striking variation between regions, it is clearly important that treatment guidelines be adopted on the basis of local surveillance and epidemiology data, be validated, and be applied with consideration for new antimicrobial drugs [4-6].

\section{The consequences of a broken beta-lactam antibiotic backbone}

A broken beta-lactam antibiotic backbone means that bacterial infections cannot be reliably treated empirically with a particular antibiotic regimen. The emergence of resistance to beta-lactam antibiotics has exerted significant pressure on the antibiotic backbone, resulting in increased morbidity, mortality, and health-care costs [28-31, 33, $34]$. Indeed, ineffective therapy can lead to many complications, including adverse events, superinfections [35], emergence of resistance [36, 37], increased ICU or hospital length of stay $[37,38]$, increased hospitalization costs [38], antibiotic-induced organ dysfunction [39], and often the need to prescribe additional antibiotics. Patients with MDR Gram-negative pneumonia may be at greater risk of death than those infected with a non-MDR organism. For example, in a recent international multicenter study in patients with $P$. aeruginosa nosocomial pneumonia, infection 
Table 1 World Health Organization-reported beta-lactam resistance rates (percentage) in common bacterial pathogens that can cause pneumonia (most recent data as reported 2013)

\begin{tabular}{|c|c|c|c|c|c|c|c|}
\hline Pathogen & Africa & Americas & $\begin{array}{l}\text { Eastern } \\
\text { Mediterranean }\end{array}$ & Europe & $\begin{array}{l}\text { South } \\
\text { East Asia }\end{array}$ & $\begin{array}{l}\text { Western } \\
\text { Pacific }\end{array}$ & WHO key points \\
\hline $\begin{array}{l}\text { Staphylococcus aureus } \\
\text { (beta-lactam resistant; } \\
\text { i.e., MRSA) }\end{array}$ & $12-80$ & $21-90$ & $10-53$ & $0.3-60$ & $10-26$ & $4-84$ & $\begin{array}{l}\text { Data on MRSA proportions among S. aureus } \\
\text { were obtained from } 44 \% \text { of member states; } \\
\text { most reported MRSA proportions exceed } \\
20 \% \text { in all WHO regions and even exceed } 80 \% \\
\text { in some reports. }\end{array}$ \\
\hline $\begin{array}{l}\text { Streptococcus pneumoniae } \\
\text { (penicillin-resistant) }\end{array}$ & $3-16$ & $0-48$ & $13-34$ & $0-61$ & $47-48$ & $17-64$ & $\begin{array}{l}\text { Data were obtained from only } 35 \% \text { of member } \\
\text { states; non-susceptibility to penicillin has been } \\
\text { detected in all WHO regions. }\end{array}$ \\
\hline $\begin{array}{l}\text { Escherichia coli } \\
\text { (resistant to third-generation } \\
\text { cephalosporins) }\end{array}$ & $2-70$ & $0-48$ & $22-63$ & $3-82$ & $16-68$ & $0-77$ & $\begin{array}{l}\text { Data on E. coli resistance to third-generation } \\
\text { cephalosporins were obtained from } 44 \% \text { of } \\
\text { member states; reports consistently disclosed } \\
\text { high resistance rates to the last-generation } \\
\text { drugs commonly used to treat serious infections. }\end{array}$ \\
\hline $\begin{array}{l}\text { Klebsiella pneumoniae } \\
\text { (resistant to third-generation } \\
\text { cephalosporins) }\end{array}$ & $8-77$ & $4-71$ & $22-50$ & $2-82$ & $34-81$ & $1-72$ & $\begin{array}{l}\text { The majority of sources reported more than } 30 \% \\
\text { resistance in K. pneumoniae to third-generation } \\
\text { cephalosporins in the sampled populations; } \\
\text { resistance proportions exceeding } 50 \% \text { were } \\
\text { reported from all WHO regions. }\end{array}$ \\
\hline $\begin{array}{l}\text { Klebsiella pneumoniae } \\
\text { (resistant to carbapenems) }\end{array}$ & $0-4$ & $0-11$ & $0-54$ & $0-68$ & $0-8$ & $0-8$ & $\begin{array}{l}\text { Rates of carbapenem resistance exceeding } 50 \% \\
\text { have been reported in some patient groups, for } \\
\text { which few, if any, alternative treatment options } \\
\text { are available. }\end{array}$ \\
\hline
\end{tabular}

with MDR strains was associated with significantly higher in-hospital mortality compared with infection with nonMDR strains $(44.7 \%$ versus $31.7 \%, P<0.001)$ [40]. However, the overall evidence on a potential association of mortality outcomes with MDR infection in patients with pneumonia is still unclear [41, 42].

\section{Current strategies to brace the antibiotic backbone}

How can the pressure on the beta-lactam antibiotic backbone be relieved? Current protective strategies include antimicrobial stewardship, the optimization of beta-lactam pharmacokinetic/pharmacodynamic (PK/PD) exposure, and the use of combination therapy with other drug classes (e.g., concurrent administration of systemic aminoglycosides).

\section{Antimicrobial stewardship}

Ideally, antibiotic stewardship in ICUs should include the rapid identification of bacterial etiology, optimization of treatment based on PK/PD characteristics of the antibiotic(s), avoidance of unnecessarily broad-spectrum antibiotics, shortening of treatment duration, and reduction in the number of patients receiving antibiotic therapy for non-infectious syndromes [43, 44]. To achieve this, ICUs should continuously collect data and adjust prescribing of beta-lactam backbone antibiotics according to their local resistance patterns [45]. Delayed appropriate therapy is consistently associated with worse outcomes [8, 46-48].
In contrast, early and appropriate empiric broad-spectrum therapy, followed by de-escalation, is generally associated with improved clinical outcomes for pneumonia [4, 49, 50]. To achieve adequate therapy, it is necessary to select the correct antibiotic(s), an optimal dose, and the correct route of administration (oral, IV, or aerosol) to ensure antibiotic penetration to the site of infection [4].

Guidelines advise that de-escalation of antibiotics be considered once information is available on the results of lower respiratory tract cultures and the patient's clinical response [4]. A meta-analysis that included three studies in patients with VAP showed that, in some patients, the use of short-course (2- to 3-day) therapy as a de-escalation approach (compared with prolonged-course therapy) was not associated with an increase in mortality, duration of mechanical ventilation, or hospital stay, particularly in those not infected with non-fermenting Gram-negative bacilli [51]. While the optimal length of antibiotic therapy for critically ill patients with VAP due to non-fermenting Gram-negative bacilli remains an unsolved issue [52], clinicians should strive to stop therapy as soon as possible.

De-escalation of therapy may be guided by scores such as the clinical pulmonary infection score (CPIS) [53]. In a randomized controlled trial (RCT), 81 patients with a CPIS of less than 6 (implying low likelihood of pneumonia) were randomly assigned to receive either standard therapy (at discretion of physician) or ciprofloxacin monotherapy, with re-evaluation at day 3; ciprofloxacin was discontinued if CPIS remained less than 6 at day 3. Mortality, length of 
ICU stay, and development of resistance did not differ significantly between treatments, actually favoring the ciprofloxacin monotherapy arm.

Biomarkers offer an alternative method to shorten duration of therapy. Several large randomized trials in critically ill patients of antibiotic duration based on falling procalcitonin levels have demonstrated that this approach can safely shorten duration without adverse consequences [54]. HAP/VAP were common types of infection in these studies.

\section{Optimizing beta-lactam pharmacokinetic/pharmacodynamic exposure}

The beta-lactam antibiotic backbone may be further supported by optimizing beta-lactam antibiotic PK/PD exposure, which could include considerations of the length of infusion and the use of appropriate loading doses. The pharmacokinetics of systemic antibiotics in critically ill patients are highly variable because of patients' physiological changes affecting drug absorption, distribution, metabolism, and elimination [55]. These changes make the correct dosing of antibiotics in these patients very challenging and can result in the delivery of sub-therapeutic or toxic drug concentrations [56, 57]. The DALI (Defining Antibiotic Levels in Intensive Care Patients) multinational ICU study was a pharmacokinetic point-prevalence study including eight systemically administered beta-lactam antibiotics [58]. Overall, $16 \%$ of patients in the study did not achieve free antibiotic concentrations sufficiently greater than the minimum inhibitory concentration (MIC) required to ensure a positive clinical outcome. Patients who had concentrations above the MIC for at least $50 \%$ or $100 \%$ of the dosing interval were more likely to have a positive clinical outcome (odds ratio 1.02 and 1.56, respectively; $P<0.03$ ) [58]. In line with this, a recent study by MacVane et al. demonstrated that, in patients with VAP caused by Gramnegative bacilli, a serum exposure greater than $53 \%$ $f \mathrm{~T}>\mathrm{MIC}$ was significantly associated with a favorable microbiological response (eradication or presumed eradication of pathogen) to anti-pseudomonal cephalosporins [59]. Similarly, a study by Muller et al. found that ceftobiprole exposure $(f \mathrm{~T}>\mathrm{MIC}$ of greater than $62 \%$ of the dosing interval) strongly correlated with microbiological eradication and clinical cure in patients with nosocomial pneumonia [60]. These and other studies (e.g., Crandon et al. [61]) emphasize the importance of considering exposure-response profiles when optimizing drug therapy in these patient groups [59-61].

One way to optimize beta-lactam antibiotic dosing may be the use of prolonged or continuous infusion, which could benefit critically ill patients with severe illness [62-64]. Continuous infusion of beta-lactams often also includes the use of a loading dose to ensure early attainment of target concentrations exceeding the MIC [63].
Currently, clinical evidence is still unclear as to whether it is better to give beta-lactam antibiotics by traditional intermittent bolus dosing or continuous infusion. Theoretically, continuous infusion of beta-lactam antibiotics should be advantageous, because it produces more sustained antibiotic concentrations above the MIC (a key measure to describe the bacterial kill characteristics of beta-lactams) [62]. A number of reports suggest that continuous infusion has clinical benefits (reviewed in [62-64]). For example, one RCT in 30 patients with severe sepsis reported that continuous administration of beta-lactam antibiotics achieved higher plasma antibiotic concentrations than intermittent administration, with associated improvements in clinical cure $(70 \%$ versus $43 \%, P=0.037$ ) [65]; however, a more recent and larger $(\mathrm{n}=432)$ trial by the same research group, again conducted in critically ill patients with severe sepsis, reported no difference in outcomes between beta-lactam antibiotic administration by continuous and intermittent infusion [66]. Indeed, two comprehensive systematic meta-analyses suggest that, overall, clinical studies in critically ill patients with acute infections have not yet conclusively demonstrated the benefits of continuous over bolus infusion [63, 64]; one of these analyses did suggest a benefit in mortality outcomes [64], whereas the other did not [63]. Clearly, further evidence, particularly from large RCTs, is needed [62-64].

Another potential strategy to help overcome the antibiotic dosing challenges in patients in the ICU may be therapeutic drug monitoring (TDM). However, a recent systematic review found that only a small number of the included studies reported that TDM may improve betalactam dosing in critically ill patients [67]. The systematic review also found little agreement between studies on the pharmacodynamic targets for optimizing antibiotic therapy. Clearly, more data are needed before any potential clinical benefits of TDM can be established $[67,68]$. However, even if clinical improvements can be conclusively demonstrated, TDM is currently not routinely available in most clinical laboratories.

\section{Other strategies}

Evidence suggests that the pressure on beta-lactams could be relieved by support from other antibiotic classes (for example, using combination therapy). A systematic review comparing outcomes of combination therapy and monotherapy for carbapenem-resistant Enterobacteriaceae (CRE)-causing respiratory infections found that combination therapy was associated with significantly lower clinical failure rates (29\% versus $67 \%, P=0.03$ ) [69].

In contrast, in a study that included 740 mechanically ventilated patients with suspected VAP, monotherapy was associated with outcomes similar to those of combination therapy [70]. Unfortunately, most high-risk patients were 
systematically excluded from this study. However, in the subgroup with MDR pathogens, combination therapy demonstrated a trend toward improved outcomes. In another study that evaluated monotherapy versus combination therapy in patients with VAP at low risk for difficult-to-treat pathogens [71], infection could be effectively managed with antibiotic monotherapy; outcomes of ICU stay, clinical response, and emergence of resistance were similar to those seen with combination therapy [71]. However, in the subgroup of patients with VAP due to Pseudomonas species, Acinetobacter species, or MDR Gram-negative bacilli $(\mathrm{n}=56)$, combination therapy demonstrated better microbiological outcomes and was associated with shorter durations of mechanical ventilation and ICU stay, although it should be noted that the study was not powered to demonstrate statistical significance for this comparison [71]. Finally, a Cochrane systematic review compared beta-lactam monotherapy with beta-lactam-aminoglycoside IV combination therapy in patients with sepsis (including patients with pneumonia) but found no change in all-cause mortality or clinical failure between mono- and combination therapy [72]. The review also found that combination therapy with an IV beta-lactam and an IV aminoglycoside carried a significant risk of nephrotoxicity (combined risk ratio in favor of monotherapy: $0.30,95 \%$ confidence interval 0.23 to 0.39$)$ [72].

\section{Future strategies to brace the antibiotic backbone}

Biomarkers are under investigation as a tool to reduce unnecessary antibiotic prescribing and further facilitate antimicrobial stewardship [73-75]. In one recent study, monitoring of procalcitonin levels (obtained upon ICU admission or after a new suspected infection, followed by a second level measurement 48 hours later) was associated with achieving significantly lower antibiotic exposure (10 versus 13 days, $P<0.003$ ) compared with using no monitoring [76]; procalcitonin monitoring was also associated with significant reductions in length of hospital stay, hospital readmission, and relapse of infection [76]. These findings are supported by several large randomized trials in critically ill patients [54].

New tools for the rapid detection of antibiotic resistance may soon aid physicians in predicting the effectiveness of empiric antibiotic therapy and enable them to quickly readjust the treatment regimen [77]. Balancing the need to confirm the pathogen(s) responsible for infection with the need to initiate prompt empiric therapy has made it difficult to select an appropriate beta-lactam antibiotic without promoting resistance. The rapid multiplex polymerase chain reaction-based Unyvero pneumonia application (UPA) assay was evaluated in 49 patients with mild-severe nosocomial pneumonia; multiple pathogens were detected in $55 \%$ of patients tested but were detected in only $8 \%$ of patients following traditional culture techniques [78]. In addition, UPA detects 13 different resistance genes, potentially permitting initial empiric treatment to be tailored within 6 hours. Although such technology is promising, further research is required to confirm that the detected organisms represent true pathogens rather than colonizing bacteria. Also, not all of the multiple resistance mechanisms in Gram-negative bacteria may be detected with polymerase chain reaction-based diagnostics. In addition, the presence of a specific resistance gene has not been conclusively demonstrated to correlate with clinical response to the associated antibiotic. This is particularly true for the various beta-lactamases that are very substrate-specific (i.e., those that mainly affect only certain beta-lactams but not others).

Another approach for optimizing antibiotic concentrations involves the delivery of high drug concentrations to the lung via aerosolization $[79,80]$. The advantages of targeted delivery include drug concentrations in the lung that greatly exceed the MIC, a strategy that could reduce the emergence of resistance, coupled with low serum concentrations that minimize systemic toxicity [79-81]. Until recently, devices used to deliver aerosols were limited by relatively poor delivery efficiency [82] and lack of an aerosol-optimized formulation (i.e., an IV formulation was given as inhalation) [81]. These limitations both impede drug delivery and contribute to adverse effects such as cough or bronchoconstriction. The development of high-efficiency nebulizers (e.g., vibrating mesh technology or adaptive nebulizers) combined with optimized formulations with particles of a size best suited to increased deposition in the lungs $(1-5 \mu \mathrm{m})[79,83]$ have the potential to enhance delivery efficiency and to overcome the limitations of previous aerosolized antibiotic treatments $[84,85]$.

Aerosolized antibiotic therapy is already administered widely in European ICUs during mechanical ventilation [86], despite limited published studies involving critically ill patients with pneumonia. A double-blind, randomized, single-center study of 24 patients at high risk of MDR organisms found that aerosolized gentamicin or amikacin every 8 hours for 2 weeks eradicated a significantly larger proportion of MDR organisms present at baseline compared with placebo $(P<0.0001)$ [87]. Furthermore, patients receiving aerosolized antibiotics had a significantly better clinical response (reduced CPIS and secretion volume) and significantly lower emergence of resistance [87]. A recent meta-analysis also supports aerosolized colistin as an adjunctive therapy to improve clinical, bacteriologic, and infection-related mortality outcomes in patients with VAP compared with IV colistin alone [88]. However, in a study in patients with VAP caused by $P$. aeruginosa, outcomes for aerosolized amikacin + ceftazidime were not significantly improved compared with IV administration of amikacin + ceftazidime (cure, $70 \%$ versus $55 \%, P=0.33$ ) [89]. 
Ongoing, prospective, randomized clinical studies with aerosolized antibiotics appear to be promising. Several options are in development: a combination amikacinfosfomycin solution delivered via a PARI eFlow inline system, which is currently in phase 2 (ClinicalTrials.gov identifier: NCT01969799); a trial with nebulized tobramycin (ClinicalTrials.gov identifier: NCT01570192), which will explore the use of nebulized tobramycin in combination with IV meropenem and an aminoglycoside (either amikacin or tobramycin); and Amikacin Inhale, an integrated drug-device combination for the delivery of specially formulated Amikacin Inhalation Solution through a Pulmonary Drug Delivery System, which is currently being investigated in two phase III studies (Clinicaltrials.gov identifiers: NCT01799993 and NCT00805168) in combination with standard of care (SOC) treatment to demonstrate clinical superiority versus SOC IV antibiotics (plus aerosol placebo) in Gram-negative pneumonia in intubated and mechanically ventilated patients in the ICU.

A phase 1 trial $(n=9)$ of combination amikacin-fosfomycin solution delivered via a PARI eFlow inline system reported that both drugs achieved high tracheal aspirate concentrations [90]. Furthermore, no adverse effects in the respiratory tract were observed. A phase 2 trial with this combination antibiotic solution using a vibrating plate nebulizer for 10 days is under way in mechanically ventilated patients with Gram-negative bacterial pneumonia.

In the completed phase 2 studies of Amikacin Inhale [91, 92], microbiologically relevant amikacin concentrations in epithelial lining fluid and tracheal aspirates were achieved after dosing of Amikacin Inhale every 12 hours. Overall, Amikacin Inhale provided high aminoglycoside concentrations in the lung (25-fold higher than reference MIC values for the Gram-negative organisms primarily responsible for pneumonia) while maintaining low serum amikacin concentrations. Data from phase 3 studies are awaited to confirm whether Amikacin Inhale in combination with SOC will improve clinical outcomes over standard IV therapies.

\section{Conclusions}

For physicians treating pneumonia in critically ill patients, the use of the beta-lactam antibiotic backbone is increasingly fraught with uncertainty. Although beta-lactam antibiotics have played an important role in the treatment of pneumonia in the ICU, resistance rates are high; in part, this has been exacerbated by widespread inappropriate prescribing of extended-spectrum beta-lactam antibiotics. The current state of resistance across many antibiotic classes is highly concerning, and the increasing difficulty of treating infections has been described as a 'major blooming public health crisis' [93].

The diligent application of antimicrobial stewardship principles, together with optimized PK/PD dosing strategies, can help preserve efficacy of the beta-lactam antibiotics-at least for the time being. However, to relieve the pressure on the beta-lactam antibiotic backbone in the long term, new approaches are urgently needed; these are likely to include the increased use of biomarkers, rapid diagnostic techniques, and new treatment approaches such as inhaled antibiotics.

\section{Abbreviations}

CPIS: Clinical pulmonary infection score; ESBL: Extended-spectrum beta-lactamase; HAP: Hospital-acquired pneumonia; HCAP: Health care-associated pneumonia; ICU: Intensive care unit; IV: Intravenous; MDR: Multi-drug-resistant; MIC: Minimum inhibitory concentration; PK/PD: Pharmacokinetic/pharmacodynamic

RCT: Randomized controlled trial; SOC: Standard of care; TDM: Therapeutic drug monitoring; UPA: Unyvero pneumonia application; VAP: Ventilator-associated pneumonia; WHO: World Health Organization.

\section{Competing interests}

TW is a member of the advisory boards of Bayer HealthCare (Leverkusen, Germany), Forest Laboratories (New York, NY, USA), Gilead Sciences (Foster City, CA, USA), and Novartis (Basel, Switzerland). He has received lecture fees from Astellas (Tokyo, Japan), Bayer Healthcare, Forest Laboratories, Gilead Sciences, Novartis, and Pfizer Inc. (New York, NY, USA) as well as basic research grants from Bayer HealthCare and Novartis. RGW is a member of data safety monitoring boards for AstraZeneca (London, UK) and Achaogen (South San Francisco, CA, USA) and acts as a consultant for Merck

(Kenilworth, NJ, USA), The Medicines Company (Parsippany, NJ, USA), Bayer HealthCare, Roche (Basel, Switzerland), Cepheid (Sunnyvale, CA, USA), and GSK (Brentford, London, UK). MB serves on scientific advisory boards for AstraZeneca, Bayer, Cubist (Lexington, MA, USA), Trius (San Diego, CA, USA), Gilead Sciences, Pfizer Inc., Merck Serono (Darmstadt, Germany), and Astellas Pharma Inc. and has received funding for travel or speaker honoraria from AstraZeneca, Gilead Sciences, Pfizer Inc., Merck Serono, Novartis, Gilead Sciences, Sumitomo (Tokyo, Japan), Teva Inc. (Petah Tikva, Israel), Astellas Pharma Inc., and Vifor Pharma (Mumbai, India).

\section{Authors' contributions}

All authors made substantial contributions to conception and design, or acquisition of data, or analysis and interpretation of data; were involved in drafting the manuscript or revising it critically for important intellectual content; and gave final approval of the version to be published. MB was accountable for all aspects of the work in ensuring that questions related to the accuracy or integrity of any part of the work are appropriately investigated and resolved.

\section{Acknowledgments}

Editorial support was provided by Highfield Communication (Oxford, UK) and funded by Bayer HealthCare.

\section{Author details}

'Santa Maria Misericordia University Hospital, Piazzale S. Maria Misericordia 15, 33100 Udine, Italy. ${ }^{2}$ Department of Respiratory Medicine, Hannover Medical School, Carl-Neuberg-Strasse 1, 30625 Hannover, Germany. ${ }^{3}$ Northwestern University Feinberg School of Medicine, 676 North St. Clair Street, Arkes 14-015, Chicago, IL 60611, USA.

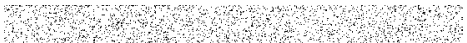

\section{References}

1. Hagihara M, Crandon JL, Nicolau DP. The efficacy and safety of antibiotic combination therapy for infections caused by Gram-positive and Gram-negative organisms. Expert Opin Drug Saf. 2010;11:221-33.

2. Spellberg B, Guidos R, Gilbert D, Bradley J, Boucher HW, Scheld WM, et al. The epidemic of antibiotic-resistant infections: a call to action for the medical community from the Infectious Diseases Society of America. Clin Infect Dis. 2008:46:155-64

3. Bell BG, Schellevis F, Stobberingh E, Goossens H, Pringle M. A systematic review and meta-analysis of the effects of antibiotic consumption on antibiotic resistance. BMC Infect Dis. 2014;14:13. 
4. American Thoracic Society; Infectious Diseases Society of America. Guidelines for the management of adults with hospital-acquired, ventilatorassociated, and healthcare-associated pneumonia. Am J Respir Crit Care Med. 2005;171:388-416.

5. Rotstein C, Evans G, Born A, Grossman R, Light RB, Magder S, et al. Clinical practice guidelines for hospital-acquired pneumonia and ventilator-associated pneumonia in adults. Can J Infect Dis Med Microbiol. 2008;19:19-53.

6. Masterton RG, Galloway A, French G, Street M, Armstrong J, Brown E, et al. Guidelines for the management of hospital-acquired pneumonia in the UK: report of the working party on hospital-acquired pneumonia of the British Society for Antimicrobial Chemotherapy. J Antimicrob Chemother. 2008;62:5-34.

7. Kollef MH. Antibiotics for the critically ill: more than just selecting appropriate initial therapy. Crit Care. 2013;17:146.

8. Iregui M, Ward S, Sherman G, Fraser VJ, Kollef MH. Clinical importance of delays in the initiation of appropriate antibiotic treatment for ventilatorassociated pneumonia. Chest. 2002;122:262-8.

9. Harbarth S, Nobre V, Pittet D. Does antibiotic selection impact patient outcome? Clin Infect Dis. 2007:44:87-93.

10. Magill SS, Edwards JR, Bamberg W, Beldavs ZG, Dumyati G, Kainer MA, et al. Multistate point-prevalence survey of healthcare-associated infections. N Engl J Med. 2014;370:1198-208.

11. Chawla R. Epidemiology, etiology, and diagnosis of hospital-acquired pneumonia and ventilator-associated pneumonia in Asian countries. Am J Infect Cont. 2008;34:593-9.

12. Barbier F, Andremont A, Wolff M, Bouadma L. Hospital-acquired pneumonia and ventilator-associated pneumonia: recent advances in epidemiology and management. Curr Opin Pulm Med. 2013;19:216-28.

13. Sopena N, Heras E, Casas I, Bechini J, Guasch I, Pedro-Botet ML, et al. Risk factors for hospital-acquired pneumonia outside the intensive care unit: a case-control study. Am J Infect Control. 2014:42:38-42.

14. Ego A, Preiser JC, Vincent JL. Impact of diagnostic criteria on the incidence of ventilator-associated pneumonia. Chest. 2015;147:347-55.

15. Stevens JP, Kachniarz B, Wright SB, Gillis J, Talmor D, Clardy P, et al. When policy gets it right: variability in US hospitals' diagnosis of ventilator-associated pneumonia. Crit Care Med. 2014;42:497-503.

16. Bekaert M, Timsit JF, Vansteelandt S, Depuydt P, Vésin A, Garrouste-Orgeas M, et al. Attributable mortality of ventilator-associated pneumonia: a reappraisal using causal analysis. Am J Respir Crit Care Med. 2011;184:1133-9.

17. Muscedere JG, Day A, Heyland DK. Mortality, attributable mortality, and clinical events as end points for clinical trials of ventilator-associated pneumonia and hospital-acquired pneumonia. Clin Infect Dis. 2010;51 Suppl 1:120-5.

18. Bonten MJM, Kollef MH, Hall JB. Risk factors for ventilator-associated pneumonia: from epidemiology to patient management. Clin Infect Dis. 2004;38:1141-9.

19. Torres A, Ferrer M, Badia JR. Treatment guidelines and outcomes of hospital-acquired and ventilator-associated pneumonia. Clin Infect Dis. 2010;51 Suppl 1:S48-53.

20. Chastre J, Fagon JY. Ventilator-associated pneumonia. Am J Respir Crit Care Med. 2002;165:867-903.

21. Timsit JF, Zahar JR, Chevret S. Attributable mortality of ventilator-associated pneumonia. Curr Opin Crit Care. 2011;17:464-71.

22. World Health Organization. Antimicrobial Resistance: Global Report on Surveillance. ISBN 978924156474 8. 2014.

23. Sader HS, Farrell DJ, Flamm RK, Jones RN. Antimicrobial susceptibility of Gram-negative organisms isolated from patients hospitalised with pneumonia in US and European hospitals: results from the SENTRY Antimicrobial Surveillance Program, 2009-2012. Int J Antimicrob Agents. 2014;43:328-34.

24. Kollef MH, Chastre J, Clavel M, Restrepo MI, Michiels B, Kaniga K, et al. A randomized trial of 7-day doripenem versus 10-day imipenem-cilastatin for ventilator-associated pneumonia. Crit Care. 2012;16:R218.

25. Jenkins SG, Fisher AC, Peterson JA, Nicholson SC, Kaniga K. Meta-analysis of doripenem vs comparators in patients with pseudomonas infections enrolled in four phase III efficacy and safety clinical trials. Curr Med Res Opin. 2009;25:3029-36

26. Chastre J, Wunderink R, Prokocimer P, Lee M, Kaniga K, Friedland I. Efficacy and safety of intravenous infusion of doripenem versus imipenem in ventilator-associated pneumonia: a multicenter, randomized study. Crit Care Med. 2008;36:1089-96

27. Zilberberg MD, Shorr AF. Prevalence of multidrug-resistant Pseudomona aeruginosa and carbapenem-resistant Enterobacteriaceae among specimens from hospitalized patients with pneumonia and bloodstream infections in the United States from 2000 to 2009. J Hosp Med. 2013;8:559-63.
28. Fraimow HS, Tsigrelis C. Antimicrobial resistance in the intensive care unit: mechanisms, epidemiology, and management of specific resistant pathogens. Crit Care Clin. 2011;27:163-205.

29. Brusselaers $N$, Vogelaers D, Blot $\mathrm{S}$. The rising problem of antimicrobial resistance in the intensive care unit. Ann Intensive Care. 2011;1:47.

30. Magiorakos A-P, Srinivasan A, Carey RB, Carmeli Y, Falagas ME, Giske CG, et al. Multidrug-resistant, extensively drug-resistant and pandrug-resistant bacteria: an international expert proposal for interim standard definitions for acquired resistance. Clin Mircobiol Infect. 2012;18:268-81.

31. Jones RN. Microbial etiologies of hospital-acquired bacterial pneumonia and ventilator-associated bacterial pneumonia. Clin Infect Dis. 2010;51 Suppl 1:S81-7.

32. Centers for Disease Control and Prevention. Antibiotic resistance threats in the United States, 2013. http://www.cdc.gov/drugresistance/threat-report2013/. Accessed 6 Aug 2015.

33. Molton JS, Tambyah PA, Ang BS, Ling ML, Fisher DA. The global spread of healthcare-associated multidrug-resistant bacteria: a perspective from Asia. Clin Infect Dis. 2013;56:1310-8.

34. Grgurich PE, Hudcova J, Lei Y, Sarwar A, Craven DE. Management and prevention of ventilator-associated pneumonia caused by multidrugresistant pathogens. Expert Rev Respir Med. 2012;6:533-55.

35. Traugott KA, Echevarria K, Maxwell P, Green K, Lewis 2nd JS. Monotherapy or combination therapy? The Pseudomonas aeruginosa conundrum. Pharmacotherapy. 2011;31:598-608.

36. Niederman MS. Use of broad-spectrum antimicrobials for the treatment of pneumonia in seriously ill patients: maximizing clinical outcomes and minimizing selection of resistant organisms. Clin Infect Dis. 2006;42:S72-81.

37. Dupont $\mathrm{H}$, Mentec $\mathrm{H}$, Sollet JP, Bleichner G. Impact of appropriateness of initial antibiotic therapy on the outcome of ventilator-associated pneumonia. Intensive Care Med. 2001:27:355-62.

38. Ott SR, Hauptmeier BM, Ernen C, Lepper PM, Nüesch E, Pletz MW, et al. Treatment failure in pneumonia: impact of antibiotic treatment and cost analysis. Eur Respir J. 2012;39:611-8.

39. Jensen JU, Hein L, Lundgren B, Bestle MH, Mohr T, Andersen MH, et al. Kidney failure related to broad-spectrum antibiotics in critically ill patients: secondary end point results from a 1200 patient randomised trial. BMJ Open. 2012;2:e000635

40. Micek ST, Wunderink RG, Kollef MH, Chen C, Rello J, Chastre J, et al. An international multicenter retrospective study of Pseudomonas aeruginosa nosocomial pneumonia: impact of multidrug resistance. Crit Care. 2015;19:219.

41. Tedja R, Nowacki A, Fraser T, Fatica C, Griffiths L, Gordon S, et al. The impact of multidrug resistance on outcomes in ventilator-associated pneumonia. Am J Infect Control. 2014:42:542-5.

42. Peña C, Gómez-Zorrilla S, Oriol I, Tubau F, Dominguez MA, Pujol M, et al. Impact of multidrug resistance on Pseudomonas aeruginosa ventilatorassociated pneumonia outcome: predictors of early and crude mortality. Eur J Clin Microbiol Infect Dis. 2013;32:413-20.

43. Katsios CM, Burry L, Nelson S, Jivraj T, Lapinsky SE, Wax RS, et al. An antimicrobial stewardship program improves antimicrobial treatment by culture site and the quality of antimicrobial prescribing in critically ill patients. Crit Care. 2012;16:R216.

44. Luyt CE, Bréchot N, Trouillet JL, Chastre J. Antibiotic stewardship in the intensive care unit. Crit Care. 2014;18:480.

45. Maechler F, Schwab F, Geffers C, Meyer E, Leistner R, Gastmeier P. Antibiotic stewardship in Germany: a cross-sectional questionnaire survey of 355 intensive care units. Infection. 2014;42:119-25.

46. Kollef MH. Broad-spectrum antimicrobials and the treatment of serious bacterial infections: getting it right up front. Clin Infect Dis. 2008;47:S3-S13.

47. Ibrahim EH, Ward S, Sherman G, Schaiff R, Fraser VJ, Kollef MH. Experience with a clinical guideline for the treatment of ventilator-associated pneumonia. Crit Care Med. 2001;29:1109-15.

48. Luna CM, Aruj P, Niederman MS, Garzón J, Violi D, Prignoni A, et al. Appropriateness and delay to initiate therapy in ventilator-associated pneumonia. Eur Respir J. 2006:27:158-64.

49. Kaki R, Elligsen M, Walker S, Simor A, Palmay L, Daneman N. Impact of antimicrobial stewardship in critical care: a systematic review. J Antimicrob Chemother. 2011;66:1223-30.

50. Masterton RG. Antibiotic de-escalation. Crit Care Clin. 2011:27:149-62.

51. Pugh R, Grant C, Cooke RPD, Dempsey G. Short-course versus prolongedcourse antibiotic therapy for hospital-acquired pneumonia in critically ill adults. Cochrane Database Syst Rev. 2011;10:CD007577. 
52. Martin-Loeches I, Torres A. A week seems to be weak: tailoring duration of antibiotic treatment in Gram-negative ventilator-associated pneumonia. Crit Care. 2013;17:106.

53. Singh N, Rogers $P$, Atwood CW, Wagener MM, Yu VL. Short-course empiric antibiotic therapy for patients with pulmonary infiltrates in the intensive care unit. A proposed solution for indiscriminate antibiotic prescription. Am J Respir Crit Care Med. 2000;162:505-11.

54. Schuetz P, Chiappa V, Briel M, Greenwald JL. Procalcitonin algorithms for antibiotic treatment decisions. Arch Intern Med. 2011;171:1322-31.

55. Smith BS, Yogaratnam D, Levasseur-Franklin KE, Forni A, Fong J. Introduction to drug pharmacokinetics in the critically ill patient. Chest. 2012;141:1327-36.

56. Blot SI, Pea F, Lipman J. The effect of pathophysiology on pharmacokinetics in the critically ill patient-concepts appraised by the example of antimicrobial agents. Adv Drug Deliv Rev. 2014;77:3-11.

57. Roberts JA, Abdul-Aziz MH, Lipman J, Mouton JW, Vinks AA, Felton TW, et al. Individualised antibiotic dosing for patients who are critically ill: challenges and potential solutions. Lancet Infect Dis. 2014;14:498-509.

58. Roberts JA, Paul SK, Akova M, Bassetti M, De Waele JJ, Dimopoulos G, et al. DALI: defining antibiotic levels in intensive care unit patients: are current $\beta$-lactam antibiotic doses sufficient for critically ill patients? Clin Infect Dis. 2014;58:1072-83.

59. MacVane SH, Kuti JL, Nicolau DP. Clinical pharmacodynamics of antipseudomonal cephalosporins in patients with ventilator-associated pneumonia. Antimicrob Agents Chemother. 2014;58:1359-64.

60. Muller AE, Punt N, Mouton JW. Exposure to Ceftobiprole is associated with microbiological eradication and clinical cure in patients with nosocomial pneumonia. Antimicrob Agents Chemother. 2014:58:2512-9.

61. Crandon JL, Bulik CC, Kuti JL, Nicolau DP. Clinical pharmacodynamics of Cefepime in patients infected with Pseudomonas aeruginosa. Antimicrob Agents Chemother. 2010;54:1111-6.

62. Abdul-Aziz MH, Dulhunty JM, Bellomo R, Lipman J, Roberts JA. Continuous beta-lactam infusion in critically ill patients: the clinical evidence. Ann Intens Care. 2012;2:37.

63. Tamma PD, Putcha N, Suh YD, Van Arendonk KJ, Rinke ML. Does prolonged $\beta$-lactam infusions improve clinical outcomes compared to intermittent infusions? A meta-analysis and systematic review of randomized, controlled trials. BMC Infect Dis. 2011;11:181.

64. Teo J, Liew Y, Lee W, Kwa AL. Prolonged infusion versus intermittent boluses of $\beta$-lactam antibiotics for treatment of acute infections: a meta-analysis. Int J Antimicrob Agents. 2014;43:403-11.

65. Dulhunty JM, Roberts JA, Davis JS, Webb SA, Bellomo R, Gomersall C, et al. Continuous infusion of beta-lactam antibiotics in severe sepsis: a multicenter double-blind, randomized controlled trial. Clin Infect Dis. 2013:56:236-44.

66. Dulhunty JM, Roberts JA, Davis JS, Webb SA, Bellomo R, Gomersall C, et al. A multicenter randomized trial of continuous versus intermittent $\beta$-lactam infusion in severe sepsis. Am J Respir Crit Care Med. 2015;192:1298-305.

67. Sime FB, Roberts MS, Peake SL, Lipman J, Roberts JA. Does beta-lactam pharmacokinetic variability in critically ill patients justify therapeutic drug monitoring? A systematic review Ann Intens Care. 2012;2:35.

68. Roberts JA, Ulldemolins M, Roberts MS, McWhinney B, Ungerer J, Paterson $\mathrm{DL}$, et al. Therapeutic drug monitoring of Beta-lactams in critically ill patients: proof of concept. Int J Antimicrob Agents. 2010;36:332-9.

69. Tzouvelekis LS, Markogiannakis A, Psichogiou M, Tassios PT, Daikos GL. Carbapenemases in Klebsiella pneumoniae and other Enterobacteriaceae: an evolving crisis of global dimensions. Clin Microbiol Rev. 2012;25:682-707.

70. Heyland DK, Dodek P, Muscedere J, Day A, Cook D. Randomized trial of combination versus monotherapy for the empiric treatment of suspected ventilator-associated pneumonia. Crit Care Med. 2008;36:737-44.

71. Hsaiky L, Murray KP, Kokoska L, Desai N, Cha R. Standard versus prolonged doripenem infusion for treatment of gram-negative infections. Ann Pharmacother. 2013;47:999-1006.

72. Paul M, Lador A, Grozinsky-Glasberg S, Leibovici L. Beta lactam antibiotic monotherapy versus beta lactam-aminoglycoside antibiotic combination therapy for sepsis. Cochrane Database Syst Rev. 2014;1:CD003344.

73. O'Brien DJ, Gould IM. Maximizing the impact of antimicrobial stewardship: the role of diagnostics, national and international efforts. Curr Opin Infect Dis. 2013;26:352-8.

74. Póvoa P, Salluh Jl. Biomarker-guided antibiotic therapy in adult critically ill patients: a critical review. Ann Intensive Care. 2012;2:32.

75. Aliberti S, Giuliani F, Ramirez J, Blasi F. How to choose the duration of antibiotic therapy in patients with pneumonia. Curr Opin Infect Dis. 2015;28:177-84.
76. Bishop BM, Bon JJ, Trienski TL, Pasquale TR, Martin BR, File Jr TM. Effect of introducing procalcitonin on antimicrobial therapy duration in patients with sepsis and/or pneumonia in the intensive care unit. Ann Pharmacother. 2014:48:577-83.

77. Jung JS, Eberl T, Sparbier K, Lange C, Kostrzewa M, Schubert S, et al. Rapid detection of antibiotic resistance based on mass spectrometry and stable isotopes. Eur J Clin Microbiol Infect Dis. 2013;33:949-55.

78. Jamal W, Al Roomi E, AbdulAziz LR, Rotimi VO. Evaluation of Cureti Unyvero, a multiplex PCR-based testing system, for rapid detection of bacteria and antibiotic resistance and impact of the assay on management of severe nosocomial pneumonia. J Clin Micro. 2014;52:2487-92.

79. Wood GC. Aerosolized antibiotics for treating hospital-acquired and ventilator-associated pneumonia. Expert Rev Anti Infect Ther. 2011;9:993-1000.

80. Palmer L. Aerosolized antibiotics in the intensive care unit. Clin Chest Med. 2011;32:559-74

81. Le J, Ashley ED, Neuhauser MM, Brown J, Gentry C, Klepser ME, et al. Consensus summary of aerosolized antimicrobial agents: application of guideline criteria. Insights from the Society of Infectious Diseases Pharmacists. Pharmacotherapy. 2010;30:562-84.

82. Le Brun PP, de Boer AH, Heijerman HG, Frijlink HW. A review of the technical aspects of drug nebulization. Pharm World Sci. 2000;22:75-81.

83. Dhand $\mathrm{R}$, Mercier E. Effective inhaled drug administration to mechanically ventilated patients. Expert Opin Drug Deliv. 2007:4:47-61.

84. Dhand R. The role of aerosolized antimicrobials in the treatment of ventilator-associated pneumonia. Respir Care. 2007;52:866-84.

85. Watts AB, McConville JT, Williams 3rd RO. Current therapies and technological advances in aqueous aerosol drug delivery. Drug Dev Ind Pharm. 2008;34:913-22.

86. Ehrmann S, Roche-Campo F, Sferrazza Papa GF, Isabey D, Brochard L, Apiou-Sbirlea G. Aerosol therapy during mechanical ventilation: an international survey. Intensive Care Med. 2013;39:1048-56.

87. Palmer LB, Smaldone GC. Reduction of bacterial resistance with inhaled antibiotics in the intensive care unit. Am J Respir Crit Care Med. 2014; 189:1225-33

88. Valachis A, Samonis G, Kofteridis DP. The role of aerosolized colistin in the treatment of ventilator-associated pneumonia: a systematic review and metaanalysis. Crit Care Med. 2015;43:527-33.

89. Lu Q, Yang J, Liu Z, Gutierrez C, Aymard G, Rouby J-J, et al. Nebulized ceftazidime and amikacin in ventilator-associated pneumonia caused by Pseudomonas aeruginosa. Am J Respir Crit Care Med. 2011;184:106-15.

90. Montgomery AB, Vallance S, Abuan T, Tservistas M, Davies A. A randomized double-blind placebo-controlled dose-escalation Phase 1 study of aerosolized amikacin and fosfomycin delivered via the PARI investigational eFlow $^{\oplus}$ inline nebulizer system in mechanically ventilated patients. J Aerosol Med Pulm Drug Del. 2014;27:441-8.

91. Niederman MS, Chastre J, Corkery K, Fink JB, Luyt CE, García MS. BAY41-6551 achieves bactericidal tracheal aspirate amikacin concentrations in mechanically ventilated patients with Gram-negative pneumonia. Intensive Care Med. 2012;38:263-71.

92. Luyt CE, Clavel M, Guntupalli K, Johannigman J, Kennedy Jl, Wood C, et al. Pharmacokinetics and lung delivery of PDDS-aerosolized amikacin (NKTR061) in intubated and mechanically ventilated patients with nosocomial pneumonia. Crit Care. 2009;13:R200.

93. Boucher H, Talbot G, Bradley J, Edwards J, Gilbert D, Rice LB, et al. Bad Bugs, no drugs: No ESKAPE! An update from the Infectious Diseases Society of America. Clin Infect Dis. 2009;48:1-12

94. Nature editorial. Available from: http://www.nature.com/nrd/journal/v6/n1/ pdf/nrd2225.pdf. Accessed 10 Dec 15. 